\title{
Relação entre conhecimento metacognitivo e desempenho em princípios de contagem
}

\author{
Relations between metacognitive knowledge and performance in \\ counting principles
}

\section{Évelin Fulginiti de Assis}

Doutoranda na Universidade Federal do Rio Grande do Sul, Porto Alegre, Rio Grande do Sul, Brasil. evelin_assis@hotmail.com - http://orcid.org/0000-0002-8542-0607

\section{Luciana Vellinho Corso}

Professora na Universidade Federal do Rio Grande do Sul, Porto Alegre, Rio Grande do Sul, Brasil. I.corso@terra.com.br - http://orcid.org/0000-0001-6384-3994

Recebido em 21 de outubro de 2018

Aprovado em 02 de outubro de 2019

Publicado em 15 de maio de 2020

\section{RESUMO}

Este artigo objetiva investigar o conhecimento metacognitivo de alunos do $1^{\circ}$ ano do Ensino Fundamental, em relação às sessões de intervenção que os sujeitos participaram, e verificar se este conhecimento corresponderia ao desempenho dos estudantes em avaliação dos princípios de contagem. Para tanto, inicialmente são discutidos estudos buscando evidenciar o conceito de metacognição e sua relação com a matemática. Este estudo contou com 60 alunos de $1^{\circ}$ ano do Ensino Fundamental, de 3 escolas públicas de Porto Alegre. A investigação do conhecimento metacognitivo ocorreu através de três perguntas realizadas, pela pesquisadora, ao final da última sessão de uma intervenção em princípios de contagem. As respostas das crianças foram categorizadas e relacionadas com o desempenho destas em avaliação dos princípios de contagem. Os resultados evidenciam que a maioria dos alunos mostrou bom conhecimento metacognitivo, demonstrando correspondência entre o que responderam à pesquisadora e o desempenho na avaliação.

Palavras-chave: Conhecimento metacognitivo. Princípios de contagem. Matemática inicial.

\section{ABSTRACT}

The objective of this article is to investigate the metacognitive knowledge of students in the first year of elementary school, related to the intervention sessions in which 
they participated, and to verify if this knowledge would correspond to their achievement in a counting principles evaluation task. In order to do that, initially studies are discussed, aiming to evidence the concept of metacognition and its relation to mathematics. This study counted with 60 students of the first year of elementary school, from 3 public schools, located in Porto Alegre. The investigation of metacognitive knowledge occurred through three questions made by the experimenter after the finalization of an intervention in counting principles. The children's answers were categorized and related to their achievement in the counting principles evaluation task. The results reveal that most of the students demonstrated good metacognitive knowledge, showing correspondence between their answers and the achievement in the evaluation task.

Keywords: Metacognitive knowledge. Counting principles. Initial mathematics.

\section{Introdução}

Este trabalho visa apresentar um estudo realizado com alunos de $1^{\circ}$ ano do Ensino Fundamental, participantes de uma pesquisa interventiva em contagem, em que foram solicitados a responder algumas questões acerca de seu desempenho nas sessões de intervenção, após o término destas. As respostas às perguntas foram relacionadas, posteriormente, com o desempenho dos estudantes em avaliação dos princípios de contagem, objetivando investigar se o conhecimento metacognitivo sobre seu desempenho estaria de acordo com o resultado da tarefa avaliativa.

Pensar sobre a própria aprendizagem é um assunto que tem sido investigado e discutido há algumas décadas (CORSO et al., 2013; FLAVELL, 1979; GAROFALO; LESTER, 1985; RIBEIRO, 2003; SCHNEIDER; ARTELT, 2010). Se aprender é um fenômeno complexo, envolvendo fatores internos e externos ao sujeito (CORSO, 2013), pensar sobre como se dá o processo de aprender pode ser considerado mais complexo ainda. Para compreender este processo, serão discutidos, a seguir, estudos que se debruçaram sobre o conceito de metacognição. 


\section{Metacognição}

Garofalo e Lester (1985) explicam que distinguir cognição de metacognição é uma tarefa difícil. Os autores percebem que a relação entre ambas consiste no fato de que a cognição está envolvida com o "fazer", enquanto a metacognição se relaciona à "escolha e planejamento do que fazer", bem como monitoramento do que está sendo feito. Além disso, quando se trata especificamente sobre metacognição, há certa dificuldade de compreendê-la, a qual pode ser atribuída ao fato de que o conceito refere-se a dois aspectos distintos, porém relacionados entre si: conhecimento e crenças sobre o fenômeno cognitivo e regulação e controle sobre ações cognitivas (GAROFALO; LESTER, 1985).

Para melhor compreender a dinâmica destes processos, pode-se recorrer ao trabalho de Flavell (1979), um dos primeiros estudiosos a se debruçar sobre a metacognição. $O$ autor em questão organizou um modelo de monitoramento cognitivo com base na ideia de que monitorar envolve quatro tipos de fenômenos: conhecimento metacognitivo, experiências metacognitivas, objetivos (ou tarefas) e ações (ou estratégias).

Conhecimento metacognitivo $(\mathrm{CM})$ refere-se ao conhecimento ou às crenças que o indivíduo possui acerca de quais fatores ou variáveis agem ou interagem, de determinadas maneiras, afetando o decorrer ou resultado de ações cognitivas. 0 CM envolve três variáveis: pessoa, tarefa e estratégias:

- A variável pessoa diz respeito ao conhecimento que o sujeito tem sobre si mesmo e sobre as outras pessoas como processadores cognitivos, podendo ser de três tipos: diferenças intraindividuais, como saber que você aprende melhor lendo do que ouvindo; diferenças interindividuais, como ter conhecimento que um de seus amigos é mais sensível do que outro; "universals of cognition" consiste em saber sobre algumas crenças universais acerca da cognição, como, por exemplo, entender que existem diversos graus de aprendizagem e tipos de compreensão; 
- A variável tarefa também possui subcategorias: a primeira envolve a informação disponível ao sujeito durante uma ação cognitiva (familiar ou não, muita ou pouca, confiável ou não, etc.), referindo-se à compreensão de quais variáveis resultam no melhor manejo da ação cognitiva e o cumprimento do objetivo colocado; a segunda subcategoria diz respeito à demanda ou objetivo da tarefa, fazendo com que o indivíduo aprenda que algumas ações são mais difíceis do que outras (por exemplo, ter conhecimento que é mais fácil lembrar a essência de uma história que as palavras exatas da história);

- A variável estratégia consiste em conhecimento, que pode ser adquirido, sobre quais estratégias são melhores ou mais efetivas para alcançar determinados objetivos.

Visando facilitar a leitura ao longo deste artigo, o termo conhecimento metacognitivo será utilizado referindo-se apenas à variável pessoa, considerando a explicação descrita anteriormente. Dessa forma, embora não seja empregado repetitivamente o termo conhecimento metacognitivo sobre si, é importante registrar que esta variável é o enfoque deste estudo.

Flavel (1979) destaca que o conhecimento metacognitivo envolve interações entre duas ou três variáveis, como por exemplo: eu (diferentemente de minha amiga) acredito que fazer resumos (em vez de apenas ler um texto) é melhor para estudar para prova (em vez de fazer um trabalho). Além disso, o autor afirma que, assim como qualquer outro tipo de conhecimento, este também pode ser impreciso, ou falhar quando precisar ser ativado ou ter muita influência ou nenhuma influência quando for ativado, etc.

As experiências metacognitivas (EM) podem ser longas ou curtas e simples ou complexas em conteúdo. A maioria tem a ver com o momento em que se está na ação cognitiva e com o tipo de progresso que está sendo feito: o sujeito pode sentir ou acreditar que esteja quase memorizando algumas instruções, ou que não está se comunicando adequadamente com outras pessoas ou que está começando 
a entender uma tarefa que será fácil de ser realizada. Segundo o autor, estas experiências possivelmente ocorrem em situações que exigem alto grau de pensamento consciente, oportunizando que sentimentos e pensamentos sobre 0 próprio pensamento surjam e, na maioria dos casos, precisem de um controle de qualidade que experiências metacognitivas podem oferecer. Este tipo de metacognição pode ter efeitos em outros aspectos metacognitivos:

- Pode levar o indivíduo a estabelecer novos objetivos ou revisar e/ou cancelar outros;

- Pode afetar o conhecimento metacognitivo ao acrescentá-lo, deletá-lo ou revisá-lo;

- Pode ativar estratégias visando objetivos cognitivos ou metacognitivos.

Os objetivos (tarefas) referem-se aos objetivos de uma ação cognitiva e as ações (estratégias) às cognições empregadas para alcançá-los. Estas classes do modelo de monitoramento cognitivo, idealizado pelo autor, são mais discutidas juntamente com o conhecimento metacognitivo e as experiências metacognitivas.

Através da ideia de Garofalo e Lester (1985) sobre os aspectos envolvidos na metacognição, mencionados anteriormente, a figura abaixo apresenta as ideias de Flavell (1979), objetivando oportunizar uma melhor visualização das questões discutidas até então.

Figura 1 - Esquema sobre metacognição

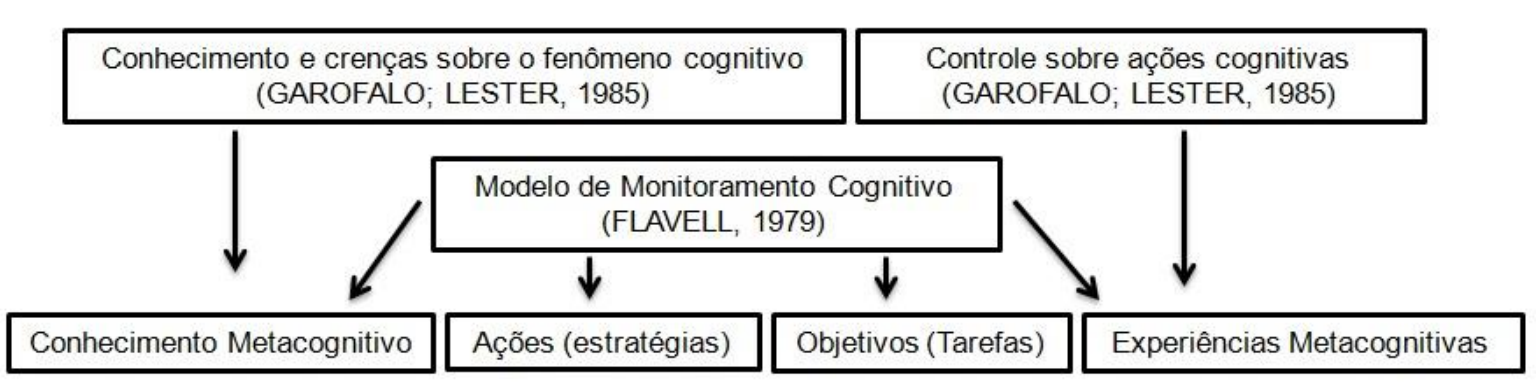

Fonte: elaborada pelas autoras. 
Reconhecendo a importância da metacognição para a aprendizagem, Ribeiro (2003) também se propõe a discutir as questões relativas à conceituação e ao papel da metacognição. A autora explora os escritos de Flavell (1979) e outros autores da área que se dedicaram a investigar o conceito mencionado, concluindo que considerar a aprendizagem numa perspectiva metacognitiva pode ser algo muito vantajoso:

- O sujeito pode desenvolver a autorregulação e autoapreciação cognitiva como formas de pensamento, de modo a ter um papel ativo na construção de seu próprio conhecimento;

- Ao destacar o papel do indivíduo na avaliação e controle cognitivos, a metacognição oportuniza novas perspectivas ao estudo de diferenças individuais no rendimento escolar;

- Ainda que seja dependente do desenvolvimento cognitivo, a metacognição possibilita à pessoa avançar no seu nível de realização ao favorecer e ser motor do próprio desenvolvimento.

De modo geral, para Ribeiro (2003), aprender a aprender, isto é, a metacognição, pode ser considerada uma capacidade muito importante da qual a aprendizagem depende. Levando em conta esta ideia e o enfoque deste trabalho, também é necessário pensar na metacognição em relação à matemática. Garofalo e Lester (1985) debruçaram-se sobre este assunto e discutiram as categorias pessoa, tarefa e estratégias do conhecimento cognitivo (FLAVELL, 1979) no âmbito matemático, destacando como o conhecimento metacognitivo pode ser compreendido nesta área:

- Conhecimento metacognitivo sobre pessoa: avaliação do sujeito sobre suas próprias capacidades e limitações em relação à matemática em geral e também sobre tópicos ou tarefas matemáticas específicas. Inclui as crenças do indivíduo acerca da natureza da habilidade matemática, da relação entre o desempenho matemático 
e o desempenho em outras áreas e dos efeitos afetivos de variáveis como motivação, ansiedade e perseverança;

- Conhecimento metacognitivo sobre tarefa: diz respeito às crenças individuais sobre a área da matemática, assim como acerca da natureza das tarefas matemáticas. Também inclui a consciência dos efeitos das características da tarefa, como conteúdo, contexto, estrutura e dificuldade;

- Conhecimento metacognitivo sobre estratégia: refere-se ao conhecimento de algoritmos e heurística, assim como a consciência do indivíduo sobre estratégias que ajudem a compreender enunciados de problemas, organizar informações ou dados, planejar soluções, executar planos e conferir resultados.

Analisando as informações apresentadas, Garofalo e Lester (1985) destacam que muitos alunos têm dificuldades com habilidades regulatórias como as mencionadas e que, para compreender o papel da metacognição no desempenho matemático, é necessário identificar uma estrutura ou modelo que a incorpore. Nesse sentido, os autores afirmam que um bom modelo deve incluir uma variedade de possíveis comportamentos, tanto cognitivos quanto de outros tipos. Além disso, também necessita destacar determinados fatores comportamentais do indivíduo que permitam identificar a presença ou ausência de ações metacognitivas. Eles citam os modelos de alguns autores, a saber, Polya (1957), Schoenfeld (1981), Sternberg $(1980,1982)$ e Luria (1973), como boas alternativas, e apresentam uma estrutura cognitiva-metacognitiva desenvolvida com base nos achados dos pesquisadores mencionados (GAROFALO; LESTER, 1985).

Tal estrutura, conforme exposto por Garofalo e Lester (1985), pode ser relacionada ao desempenho em uma variedade de tarefas matemáticas, não apenas resolução de problemas. Além disso, especifica pontos importantes em que decisões metacognitivas podem influenciar ações cognitivas, contando com quatro categorias de atividades: orientação, organização, execução e verificação. 
- A orientação refere-se ao comportamento estratégico para avaliar e compreender um problema, incluindo compreensão de estratégias, análise de informações e condições, avaliação da familiaridade com a tarefa, representação inicial e subsequente, avaliação do nível de dificuldade e chances de sucesso;

- A organização diz respeito ao planejamento do comportamento e escolha de ações, englobando identificação de objetivos e subobjetivos, planejamento global e planejamento local;

- A execução consiste na regulação do comportamento para estar de acordo com os planos, contando com desempenho em ações locais, monitoramento do progresso de planos globais e locais e decisões relacionadas à velocidade, precisão;

- A verificação corresponde à avaliação das decisões tomadas e dos resultados dos planos realizados, como avaliação da orientação e organização e avaliação da execução (GAROFALO; LESTER, 1985).

Os autores afirmam que a estrutura desenvolvida tem como objetivo atuar como um instrumento para analisar a metacognição no desempenho matemático. Eles indicam que pode ser utilizada para a realização de pesquisas, no sentido de selecionar tarefas avaliativas e desenvolver entrevistas, bem como interpretar dados e organizar análises.

\section{Metacognição e matemática}

Mesmo que a metacognição venha sendo estudada há algumas décadas, ainda há muito a ser explorado e investigado. A seguir serão apresentados alguns estudos empíricos que, de alguma maneira, analisaram o papel da metacognição em relação ao desempenho matemático.

Eflkides e Tsiora (2002) desenvolveram uma pesquisa com o objetivo de identificar o papel das experiências metacognitivas (EM) na formação do 
autoconceito no domínio matemático e na autorregulação. Ainda que estes conceitos não tenham sido discutidos até então no presente trabalho, é válido analisar os resultados relativos às EM. As autoras discorrem sobre as questões teóricas envolvidas no estudo e, no que se refere às experiências, explicam que sentimentos de dificuldade, confiança, satisfação, estimativa de esforço e estimativa de solução são bons exemplos de sentimentos metacognitivos relacionados às EM.

Foi realizado um estudo longitudinal com a participação de 163 alunos do $5^{\circ}$ e 6ํaㅅ ano do Ensino Fundamental. Dentre as medidas de avaliação utilizadas, é válido ressaltar que as experiências metacognitivas foram investigadas através de um questionário administrado antes e depois da resolução de cada tarefa. As questões consistiam em: "o quão difícil você acha que esta tarefa foi?", "o quanto de esforço você acha que precisa (precisou) despender para resolver este problema?" e "o quão corretamente você acha que irá resolver (resolveu) este problema?". As respostas correspondiam a uma escala de 4 pontos.

Os resultados sugeriram que as experiências metacognitivas, como sentimento de dificuldade, estimativa de solução correta e estimativa de esforço, formam uma ligação indispensável no ciclo da autorregulação. As EM são a interface entre si e tarefa por meio de duas maneiras: primeiro, com base em desempenhos anteriores e autoconceitos relacionados ao domínio de determinada tarefa, as experiências conscientizam o sujeito de sua capacidade para lidar com a atividade em questão e da quantidade de esforço necessária para isso; em segundo lugar, a partir do monitoramento do processamento cognitivo, desempenho e esforço, as EM informam o indivíduo sobre os aspectos que qualificarão o resultado da tarefa e suas implicações para o autoconceito (EFKLIDES; TSIORA, 2002).

Aunola et al. (2004) realizaram um estudo sobre a dinâmica de desenvolvimento do desempenho matemático da Educação Infantil ao $2^{\circ}$ ano do Ensino Fundamental (EF), buscando compreender qual o papel de alguns "antecedentes cognitivos" neste processo. Dentre estes, as autoras destacam a contagem, o conhecimento metacognitivo, recursos atencionais e compreensão auditiva. A pesquisa contou com 194 crianças, com idades entre 5 e 6 anos, avaliadas da Educação Infantil ao $2^{\circ}$ ano do EF. Para examinar o desempenho 
matemático, foi utilizada a medida Diagnostic Test for Basic Mathematical Concepts e, dentre as tarefas avaliativas de antecedentes cognitivos, é importante destacar The Metacognitive Knowledge Test - MKT, parte de um instrumento mais abrangente denominado Diagnostic Tests for Metacognitions and Mathematics.

$O$ teste utilizado compreende quatro tarefas em que as crianças observam dois ou três cartões que contêm um menino ou uma menina usando uma abordagem diferente para aprender algo. Após observarem os cartões, os alunos deveriam apontar para aquele em que o indivíduo mostrado estivesse utilizando a estratégia mais efetiva para aprender. Depois dos sujeitos escolherem um cartão, deveriam justificar sua escolha, tendo suas respostas escritas pelo pesquisador.

Os achados do estudo apontaram o importante papel da metacognição. Foi evidenciado que o nível de conhecimento metacognitivo tem relação com o nível de desempenho matemático dos alunos, demonstrando que, quanto maior o nível de CM no início da Educação Infantil, maior o nível inicial do desempenho matemático. Não obstante, o conhecimento metacognitivo não foi preditor da performance matemática, sugerindo que embora boas estratégias de aprendizagem, como as metacognitivas, sejam relevantes para o uso de conhecimentos e habilidades já adquiridas, talvez não tenham o mesmo efeito na aquisição de novas habilidades (AUNOLA et al., 2004).

Özsoy (2011) realizou uma investigação sobre a relação entre metacognição e desempenho matemático. $O$ autor buscou avaliar o conhecimento metacognitivo e habilidades metacognitivas de 242 alunos de $5^{\circ}$ ano do Ensino Fundamental e investigar a relação entre seu desempenho e metacognição. Os instrumentos utilizados foram Mathematics Achievement Test - MAT para desempenho matemático e uma versão adaptada do Metacognitive Skills and Knowledge Assessment - MAS. Este instrumento avalia dois componentes metacognitivos, conhecimento e habilidades, bem como sete parâmetros metacognitivos: conhecimento declarativo, procedural e condicional; predição; planejamento; monitoramento; habilidades de avaliação.

Os resultados evidenciaram uma relação positiva e significativa entre metacognição e desempenho matemático, indicando que uma boa performance em 
matemática está ligada à uma habilidade metacognitiva adequada. A pesquisa também mostrou que há relação significativa entre cada parâmetro metacognitivo e o desempenho matemático (ÖZSOY, 2011).

Throndsen (2011), em estudo longitudinal, examinou, dentre outros objetivos, as relações entre as habilidades matemáticas básicas de crianças da Educação Infantil e o uso de estratégias matemáticas, competências metacognitivas e crenças motivacionais. Para tanto, a autora contou com a participação de 27 alunos do $2^{\circ}$ ano do Ensino Fundamental, avaliando-os em diferentes medidas de desempenho, estratégias, metacognição e motivação.

Em relação à metacognição, foram avaliados o conhecimento metacognitivo e a regulação através de entrevistas estruturadas que eram realizadas após a resolução de problema aritmético. As perguntas eram relacionadas ao conhecimento procedural (pode me dizer como você resolveu o problema?), conhecimento declarativo (pode me dizer outras maneiras de resolver este problema?) e conhecimento situacional (por que você resolveu o problema desta maneira? Você pode me falar outro problema que resolveria do mesmo jeito?).

Os dados encontrados indicam que uma boa performance aritmética está relacionada não apenas com o uso avançado de estratégias matemáticas, como também, dentre outros aspectos, com as competências metacognitivas de domínio específico. Do ponto de vista da autorregulação, o conhecimento metacognitivo desempenha um papel importante no uso de estratégias matemáticas pela criança e na performance acadêmica (THRONDSEN, 2011).

Efklides e Vlachopoulos (2012) realizaram uma pesquisa acerca da medida de conhecimento metacognitivo sobre si, tarefa e estratégia em matemática. Os autores desenvolveram um questionário, Metacognitive Knowledge in Mathematics Questionnaire (MKMQ) e, dentre outros objetivos, investigaram as relações entre CM em matemática, habilidades matemáticas e autoconceito matemático.

A pesquisa contou com uma amostra de 311 alunos dos anos finais do Ensino Fundamental ( $7^{\circ}, 8^{\circ}$ e $9^{\circ}$ anos) e outra de 214 universitários que cursavam Psicologia. Os sujeitos foram avaliados em tarefas relacionadas às habilidades matemáticas, autoconceito matemático, atividades matemáticas, experiências 
metacognitivas e no instrumento desenvolvido pelo primeiro autor. Este instrumento consistia em sete subescalas: conhecimento metacognitivo sobre si - facilidade e fluência; conhecimento metacognitivo sobre si - dificuldade e falta de fluência; conhecimento metacognitivo sobre tarefa - demandas fáceis e baixas; conhecimento metacognitivo sobre tarefa - demandas altas e difíceis; conhecimento metacognitivo sobre estratégias - cognitivo e metacognitivo; conhecimento metacognitivo sobre estratégias - estratégias para melhorar a competência; conhecimento metacognitivo sobre estratégias - estratégias de avoidance ${ }^{2}$, no sentido de evitar determinadas escolhas.

Os resultados demonstraram que a medida organizada pelo autor consiste em sete fatores relacionados, sugerindo que cada um destes captura um aspecto diferente da metacognição em matemática. Foi evidenciado que o conhecimento metacognitivo sobre si ocorre em duas dimensões: (1) facilidade e fluência significa habilidade, enquanto (2) dificuldade e falta de fluência, falta de habilidade. $O$ conhecimento metacognitivo sobre tarefa envolve demandas altas ou baixas, mas, diferentemente das duas dimensões de CM sobre pessoa, ambas pertencem à mesma dimensão (demandas). Estes achados indicam que os sujeitos selecionam estratégias com base nas representações de si mesmos, como processadores fluentes ou não (CM sobre si), e, posteriormente, de acordo com suas crenças sobre a demanda da tarefa (CM sobre tarefa). No que diz respeito ao conhecimento metacognitivo sobre estratégias, foi evidenciado que a consciência sobre fluência ou falta de fluência está associada ao uso de estratégias de avoidance, em vez de estratégias construtivas para superar dificuldades (EFKLIDES; VLACHOPOULOS, 2012).

As pesquisas descritas até o momento apontam para o importante papel da metacognição na aprendizagem. Dentre os achados gerais, pode-se destacar que: as experiências metacognitivas são uma ligação indispensável no ciclo de autorregulação (EFKLIDES; TSIORA, 2002); indivíduos que possuem um alto nível de conhecimento cognitivo também apresentam alto nível de habilidade matemática (AUNOLA et al., 2004); alunos com boa performance matemática também possuem boa performance metacognitiva (ÖZSOY, 2011); bom desempenho em aritmética está relacionado não apenas ao uso de estratégias matemáticas, como também às 
competências metacognitivas (THRONDSEN, 2011); e o conhecimento metacognitivo sobre si é diferente do $\mathrm{CM}$ sobre tarefa e estratégias, com implicações distintas em cada um (EFKLIDES; VLACHOPOULOS, 2012).

Considerando o que foi apresentado até o momento e com o intuito de contribuir para as discussões nesta área, será apresentado, a seguir, um estudo com enfoque na metacognição. Os objetivos deste foram investigar o conhecimento metacognitivo de alunos do $1^{\circ}$ ano do Ensino Fundamental em relação às sessões de intervenção em princípios de contagem que participaram e verificar se o $\mathrm{CM}$ dos alunos em questão corresponde ao desempenho destes em avaliação dos princípios de contagem. Esperava-se que os alunos associariam suas percepções acerca de sua performance nas sessões de intervenção de acordo com a facilidade/dificuldade enfrentada e que o desempenho na tarefa avaliativa sobre princípios de contagem refletiria as percepções dos alunos.

\section{Método}

\section{Amostra}

A amostra contou com 60 alunos, entre 6 e 7 anos de idade (30 meninas e 30 meninos), participantes de um estudo maior, experimental, intitulado "Intervenção em princípios de contagem para alunos do $1^{\circ}$ ano do Ensino Fundamental". Estes sujeitos, provenientes de 10 turmas de 3 escolas públicas de Porto Alegre, compuseram o grupo experimental da pesquisa em questão, o qual recebeu 4 sessões de intervenção em princípios de contagem. Todos os envolvidos foram autorizados a participar do estudo através do Termo de Consentimento Livre e Esclarecido.

\section{Instrumentos}

\section{Conhecimento metacognitivo sobre si}

$\mathrm{Na}$ etapa 2 do estudo experimental mencionado anteriormente, as crianças receberam 4 sessões de intervenção em princípios de contagem, com duração de 
20-35 minutos, em grupos de no máximo 5 alunos. No final da última sessão, a pesquisadora relembrou o que havia sido realizado em cada sessão:

- Na primeira sessão, conversou-se sobre os números. Depois, estes foram procurados em jornais e revistas. Por fim, jogou-se o jogo "qual está faltando";

- $\mathrm{Na}$ segunda sessão, trabalhou-se com desenhos contendo "bolinhas", as quais foram contadas com o uso de pequenas fichas;

- Na terceira sessão, jogou-se dois jogos de tabuleiro: "jogo das cores" e "duas mãos";

- Na quarta e última sessão, o jogo utilizado foi "bingo".

Após esta retomada, os alunos foram solicitados a relatar (a) algo novo que foi aprendido, (b) algo que acharam fácil e (c) algo que acharam difícil, com suas respostas sendo registradas por escrito pela pesquisadora para posterior análise.

\section{Avaliação dos princípios de contagem}

O instrumento utilizado avaliou a consolidação dos princípios de contagem (GELMAN; GALLISTEL, 1978) pelas crianças. Para cada princípio, uma ou mais perguntas são realizadas, com as respostas sendo classificadas em "sim" se a criança demonstrar domínio, "em construção" se apresentar dúvidas ou respostas pouco consistentes e "não" se não evidenciar nenhuma compreensão.

Tabela 1: Avaliação dos princípios de contagem

\begin{tabular}{|c|c|}
\hline Ordem estável & É perguntado à criança “até quando sabes contar?", seguido da \\
solicitação que conte.
\end{tabular}

(continua) 
Tabela 1: Avaliação dos princípios de contagem

\begin{tabular}{|c|c|}
\hline Cardinalidade & $\begin{array}{c}\text { Ao final da contagem de um grupo de 15 elementos, as crianças são } \\
\text { solicitadas a responder "quantos têm ao todo? Podes me dar 10 } \\
\text { fichas?". }\end{array}$ \\
\hline Abstração & Pergunta-se: "se estivesses contando 15 balas, contarias da mesma \\
forma que contaste as fichas?".
\end{tabular}

Fonte: elaborada pelas autoras.

(conclusão)

Foram estabelecidos determinados critérios para registrar as respostas das crianças, visto que as perguntas 2, 3 e 5 possuem mais de uma solicitação. Foi estabelecido que a resposta seria "sim" caso o aluno respondesse corretamente todos os questionamentos de cada pergunta; "em construção" se descumprisse com alguma das solicitações; "não" caso deixasse de responder corretamente a todas as solicitações.

\section{Procedimentos}

A pesquisa de intervenção ocorreu entre os meses de setembro e dezembro de 2017, contando com três etapas: (1) pré-teste, (2) intervenção e (3) pós-teste. Neste artigo, serão utilizados dados das etapas 2 e 3, somente dos estudantes do grupo experimental.

$\mathrm{Na}$ etapa 2, conforme mencionado anteriormente, as crianças receberam 4 sessões de intervenção em princípios de contagem. Ao final da última sessão, a pesquisadora relembrou o que havia sido realizado em cada sessão e realizou as perguntas já citadas. Na etapa 3, pós-teste, todos os alunos foram avaliados na tarefa de avaliação dos princípios de contagem. 


\section{Análise de dados}

Foram realizados dois testes nas análises: binomial e qui-quadrado. Ambos foram feitos com o objetivo de investigar se as respostas estariam igualmente distribuídas entre os constructos de cada princípio, comparando-as. Há diferença significativa entre os constructos quando o $p$-valor encontrado é menor que o nível de significância adotado $(0,05)$. $O$ teste binomial foi adotado quando havia alunos em dois constructos e o qui-quadrado quando havia alunos distribuídos em três constructos. Quando todos os alunos pertenciam ao mesmo constructo, não houve necessidade de comparação.

\section{Resultados}

Serão expostos os resultados considerando os objetivos estabelecidos anteriormente. Os achados relativos à cada pergunta feita pela pesquisadora serão apresentados através de duas tabelas: a primeira demonstra as respostas dadas pelas crianças, enquanto a segunda contém as análises realizadas.

Tabela 2 - Respostas à pergunta "o que aprendeu?"

\begin{tabular}{|l|l|l|}
\hline O que aprendeu? & Respostas & $\%$ \\
\hline Contar do jeito certo & 23 & 44,23 \\
\hline Jogar jogo(s) de tabuleiro & 8 & 15,38 \\
\hline Qual está faltando? & 6 & 11,54 \\
\hline Bingo & 4 & 7,69 \\
\hline Pode começar a contar por onde quiser & 4 & 7,69 \\
\hline Contar elementos & 3 & 5,77 \\
\hline Achar números no jornal & 2 & 3,85 \\
\hline Contar com fichinhas & 2 & 3,85 \\
\hline Total & 52 & 100,00 \\
\hline
\end{tabular}

Fonte: elaborada pelas autoras. Legenda: "o que aprendeu" - categorias de respostas dadas pelas crianças; "respostas" - número de vezes que esta resposta foi dada; "\%" - porcentagem do ํㅡ de respostas. 
A Tabela 1 apresenta as respostas fornecidas pelas crianças à pergunta "o que aprendeu?". Há apenas 52 respostas computadas porque algumas crianças relataram aprendizagens não relacionadas às tarefas da intervenção, sendo retiradas da análise por conta disso. As categorias correspondem às tarefas realizadas durante o período de intervenção e cada uma pode ser associada a um ou mais princípios de contagem. "Contar do jeito certo" envolve os cinco princípios, uma vez que, ao longo dos encontros da pesquisadora com os alunos, foram discutidos os aspectos relevantes para realizar a contagem corretamente. "Jogar jogo(s) de tabuleiro" também envolve os cinco princípios, uma vez que os dois jogos utilizados na intervenção foram desenvolvidos com este propósito. "Qual está faltando" é uma tarefa que teve como foco os princípios ordem estável, correspondência termo a termo e cardinalidade. "Bingo" é um jogo que exigiu dos alunos a utilização dos cinco princípios. "Pode começar a contar por onde quiser" remete ao princípio da irrelevância da ordem. "Contar elementos" diz respeito ao princípio da abstração, pois os estudantes constataram que não se conta apenas números, como também outros elementos. "Achar números no jornal" foi uma das primeiras tarefas realizadas na intervenção e envolveu os princípios ordem estável, correspondência termo a termo e cardinalidade. "Contar com fichinhas" enfatiza o princípio da correspondência termo a termo.

A tabela abaixo foi organizada da seguinte maneira: a primeira coluna corresponde às categorias discutidas anteriormente; a segunda coluna possui as letras representantes de cada princípio, sendo importante ressaltar que o princípio ordem estável não foi considerado nesta análise porque todas as crianças sabiam contar até determinada quantidade e, portanto, as atividades $A, B, C$ e D correspondem, respectivamente, aos princípios correspondência termo a termo, cardinalidade, abstração e irrelevância da ordem; as colunas seguintes representam cada um dos princípios e os constructos das crianças, representados pelas letras "S" para sim, "EC" para em construção e "N" para não; a coluna p-valor representa o valor descritivo amostral para os testes estatísticos de comparação. Para melhor visualização das tabelas, foram incluídas apenas as atividades (princípios) que contaram com crianças distribuídas em dois ou três constructos. Atividades que 
apresentaram apenas um constructo ou apenas dois constructos, mas com poucos sujeitos em um destes, foram retiradas da tabela. De qualquer modo, todos os constructos foram discutidos e analisados na interpretação dos dados, na medida em que as categorias foram mencionadas.

Tabela 3 - Relação entre respostas e constructos (o que aprendeu?)

\begin{tabular}{|c|c|c|c|c|c|c|c|c|c|c|}
\hline \multirow{2}{*}{ O que aprendeu? } & \multirow{2}{*}{ Princípio } & \multirow{2}{*}{ Respostas } & \multicolumn{3}{|c|}{ Atividade $\mathrm{A}$} & \multirow[b]{2}{*}{ p-valor } & \multicolumn{3}{|c|}{ Atividade D } & \multirow[b]{2}{*}{$p$-valor } \\
\hline & & & $\mathrm{N}$ & $\mathrm{EC}$ & $\mathrm{S}$ & & $\mathrm{N}$ & $\mathrm{EC}$ & $\mathrm{S}$ & \\
\hline \multirow{2}{*}{$\begin{array}{l}\text { contar do jeito } \\
\text { certo }\end{array}$} & \multirow{2}{*}{$A, B, C, D$} & 23 & & 11 & 12 & 1,000 & 3 & 7 & 13 & 0,0367 \\
\hline & & & $0 \%$ & $47,83 \%$ & $52,17 \%$ & & $13,04 \%$ & $30,43 \%$ & $56,52 \%$ & \\
\hline \multirow{2}{*}{$\begin{array}{l}\text { jogar jogo(s) de } \\
\text { tabuleiro }\end{array}$} & \multirow{2}{*}{$A, B, C, D$} & 8 & & 5 & 3 & 0,7266 & & 6 & 2 & 0,2891 \\
\hline & & & $0 \%$ & $62,50 \%$ & $37,50 \%$ & & $0 \%$ & $75,00 \%$ & $25,00 \%$ & \\
\hline \multirow{2}{*}{ bingo } & \multirow{2}{*}{$A, B, C, D$} & 4 & & 1 & 3 & 0,6250 & 1 & 1 & 2 & 0,7788 \\
\hline & & & $0 \%$ & $25,00 \%$ & $75,00 \%$ & & $25,00 \%$ & $25,00 \%$ & $50,00 \%$ & \\
\hline qual está & \multirow{2}{*}{$A, B$} & 6 & & 1 & 5 & 0,2188 & & 2 & 4 & 0,6875 \\
\hline faltando? & & & $0 \%$ & $16,67 \%$ & $83,33 \%$ & & & $50,00 \%$ & $100,00 \%$ & \\
\hline achar números & \multirow{2}{*}{$A, B$} & 2 & & & 2 & & 1 & $0 \%$ & 1 & 1,000 \\
\hline no jornal & & & & & $100 \%$ & & $50,00 \%$ & & $50,00 \%$ & \\
\hline contar com & \multirow{2}{*}{ A } & 2 & & & 2 & & & & 2 & \\
\hline fichinhas & & & & & $100 \%$ & & & & $100 \%$ & \\
\hline contar & \multirow{2}{*}{$\mathrm{C}$} & 3 & & 1 & 2 & 1,000 & 1 & 1 & 1 & 1,000 \\
\hline elementos & & & $0 \%$ & $33,33 \%$ & $66,67 \%$ & & $33,33 \%$ & $33,33 \%$ & $33,33 \%$ & \\
\hline pode começar a & \multirow{2}{*}{ D } & 4 & & & 4 & & & 2 & 2 & 1,000 \\
\hline contar por onde & & & & & $100 \%$ & & $0 \%$ & $50,00 \%$ & $50,00 \%$ & \\
\hline
\end{tabular}

Fonte: elaborada pelas autoras. Legenda: "o que foi difícil" - pergunta realizada pela pesquisadora; "princípios" - princípios de contagem representados pelas letras; "atividade A" - correspondência termo a termo"; "atividade D" - irrelevância da ordem; "N"- princípio não construído; "EC" - princípio em construção; "S"- sim, princípio consolidado; sig - significância estatística.

Para melhor interpretar as análises realizadas, a tabela apresenta apenas os princípios que foram foco de cada categoria, conforme discutido acima. Procurouse testar se, além dos casos em que todos os alunos têm apenas um constructo, havia diferença entre as proporções de alunos de cada constructo para uma mesma resposta em cada atividade. Dessa forma, os achados indicam que apenas a categoria "contar do jeito certo" das atividades C e D tiveram proporções diferentes, conforme indicado pelo $p$-valor $<0,05$, o que evidencia diferença significativa da quantidade de respostas entre os constructos. A resposta "contar do jeito certo" de 
ISSN: 1984-6444 | http://dx.doi.org/10.5902/1984644435294

constructo $S$ foi prevalente em comparação aos demais constructos nas atividades B (100\%), C (95,65\%) e D (56,52\%).

Tabela 4 - Respostas à pergunta "o que foi fácil?"

\begin{tabular}{|l|c|c|}
\hline O que foi fácil? & Respostas & $\%$ \\
\hline Bingo & 16 & 30,77 \\
\hline Contar bolinhas & 11 & 21,15 \\
\hline Tudo & 9 & 17,31 \\
\hline Jogo das cores & 8 & 15,38 \\
\hline Jogo das mãos & 8 & 15,38 \\
\hline Qual está faltando & 7 & 13,46 \\
\hline Contar os números & 2 & 3,85 \\
\hline Procurar no no jornal & 2 & 3,85 \\
\hline Total & 63 & $100,00 \%$ \\
\hline
\end{tabular}

Fonte: elaborada pelas autoras. Legenda: "o que aprendeu" - categorias de respostas dadas pelas crianças; "respostas" - número de vezes que esta resposta foi dada; "\%" - porcentagem do ㄲo de respostas.

Em relação à pergunta "o que foi fácil", as categorias organizadas na tabela acima correspondem às respostas das crianças. No que diz respeito aos princípios foco de cada uma, a maioria já foi discutida anteriormente, mas algumas são informações novas e serão explicadas a seguir. "Contar bolinhas" teve como foco os princípios ordem estável, correspondência termo a termo, cardinalidade e irrelevância da ordem. "Jogo das cores" e "jogo das mãos" são a categoria da tabela anterior, "jogar jogo(s) de tabuleiro" divida em subcategorias, envolvendo, também, todos os princípios de contagem. "Contar os números" também tem como foco os cinco princípios e "procurar os números no jornal" teve enfoque sobre os princípios ordem estável, correspondência termo a termo e cardinalidade. 
ISSN: 1984-6444 | http://dx.doi.org/10.5902/1984644435294

Tabela 5 - Relação entre respostas e constructos (o que foi fácil?)

\begin{tabular}{|c|c|c|c|c|c|c|c|c|c|c|}
\hline \multirow{2}{*}{ O que foi fácil? } & \multirow{2}{*}{ Princípio } & \multirow{2}{*}{ Respostas } & \multicolumn{3}{|c|}{ Atividade A } & \multirow[b]{2}{*}{ sig } & \multicolumn{3}{|c|}{ Atividade D } & \multirow[b]{2}{*}{ sig } \\
\hline & & & $\mathrm{N}$ & $E C$ & $\mathrm{~S}$ & & $\mathrm{~N}$ & EC & $S$ & \\
\hline \multirow{2}{*}{ bingo } & \multirow{2}{*}{$A, B, C, D$} & 16 & & 7 & 9 & 0,8036 & & 8 & 8 & 1,000 \\
\hline & & & $0 \%$ & $43,75 \%$ & $56,25 \%$ & & $0 \%$ & $50,00 \%$ & $50,00 \%$ & \\
\hline \multirow{2}{*}{ tudo } & \multirow{2}{*}{$A, B, C, D$} & 9 & & 4 & 5 & 1,000 & & 5 & 4 & 1,000 \\
\hline & & & $0 \%$ & $44,44 \%$ & $55,56 \%$ & & $0 \%$ & $55,56 \%$ & $44,44 \%$ & \\
\hline \multirow{2}{*}{ jogo das cores } & \multirow{2}{*}{$A, B, C, D$} & 8 & & 3 & 5 & 0,7266 & 1 & 4 & 3 & 0,4169 \\
\hline & & & $0 \%$ & $37,50 \%$ & $62,50 \%$ & & $12,50 \%$ & $50,00 \%$ & $37,50 \%$ & \\
\hline \multirow{2}{*}{ jogo das mãos } & \multirow{2}{*}{$A, B, C, D$} & 8 & & 2 & 6 & 0,2891 & 2 & 1 & 5 & 0,1969 \\
\hline & & & $0 \%$ & $25,00 \%$ & $75,00 \%$ & & $25,00 \%$ & $12,50 \%$ & $62,50 \%$ & \\
\hline \multirow{2}{*}{$\begin{array}{l}\text { contar os } \\
\text { números }\end{array}$} & \multirow{2}{*}{$A, B, C, D$} & 2 & & & 2 & & & & 2 & \\
\hline & & & & & $100 \%$ & & & & $100 \%$ & \\
\hline \multirow{2}{*}{ contar bolinhas } & \multirow{2}{*}{$A, B, D$} & 11 & & 2 & 9 & 0,0654 & 1 & 2 & 8 & 0,0201 \\
\hline & & & $0 \%$ & $18,18 \%$ & $81,82 \%$ & & $9,09 \%$ & $18,18 \%$ & $72,73 \%$ & \\
\hline \multirow{4}{*}{$\begin{array}{l}\text { qual está } \\
\text { faltando } \\
\text { procurar no no } \\
\text { jornal }\end{array}$} & \multirow{2}{*}{$A, B$} & 7 & & 2 & 5 & 0,4531 & 2 & 1 & 4 & 0,3679 \\
\hline & & & $0 \%$ & $28,57 \%$ & $71,43 \%$ & & $28,57 \%$ & $14,29 \%$ & $57,14 \%$ & \\
\hline & \multirow{2}{*}{ A, B } & 2 & & 1 & 1 & 1,000 & & 2 & & \\
\hline & & & $0 \%$ & $50,00 \%$ & $50,00 \%$ & & & $100 \%$ & & \\
\hline
\end{tabular}

Fonte: elaborada pelas autoras. Legenda: "o que foi difícil" - pergunta realizada pela pesquisadora; "princípios" - princípios de contagem representados pelas letras; "atividade A" - correspondência termo a termo"; "atividade D" - irrelevância da ordem; "N"- princípio não construído; "EC" - princípio em construção; "S"- sim, princípio consolidado; sig - significância estatística.

Nesta tabela, as atividades B e C não foram incluídas por conta dos critérios mencionados anteriormente. A tabela mostra que, de fato, a maioria estava construindo ou dominava os princípios, com apenas 1 caso demonstrando não consolidação do princípio abstração. Embora quase todo o grupo tenha relatado facilidade e evidenciado bom desempenho, é importante atentar para o caso do indivíduo que obteve constructo "N" em parte da avaliação, sendo minoria significativa comparada a "S" ( $p$-valor 0,0391).

Seguindo a tendência de outras respostas, a categoria "contar bolinhas" também evidenciou que a maioria do grupo relatou facilidade e de fato apresentou constructos "EC" e "S" na avaliação, com apenas 1 caso divergindo no princípio da irrelevância da ordem. Mesmo assim, dos 11 alunos correspondentes a essa categoria, a maioria com constructo "S" (8) foi significativa (p-valor de 0,02 ). 
ISSN: 1984-6444 | http://dx.doi.org/10.5902/1984644435294

Tabela 6 - Respostas à pergunta "o que foi difícil?"

\begin{tabular}{|l|c|c|}
\hline O que foi difícil? & Respostas & $\%$ \\
\hline Nada & 24 & 46,15 \\
\hline Bingo & 12 & 23,08 \\
\hline Jogo das cores & 7 & 13,46 \\
\hline Qual está faltando & 7 & 13,46 \\
\hline Jogo das mãos & 6 & 11,54 \\
\hline Contar bolinhas & 4 & 7,69 \\
\hline Achar no no jornal & 3 & 5,77 \\
\hline Total & 63 & 100,00 \\
\hline
\end{tabular}

Fonte: elaborada pelas autoras. Legenda: "o que aprendeu" - categorias de respostas dadas pelas crianças; "respostas" - número de vezes que esta resposta foi dada; "\%"- porcentagem do no de respostas.

As respostas (categorias) apresentadas à pergunta "o que foi difícil?" são semelhantes às categorias das outras questões. A única resposta diferente é "nada", relatada por 24 crianças e, assim como a categoria "tudo" da questão "o que foi fácil?", é uma afirmação mais generalizada.

Tabela 7 - Relação entre respostas e constructos (o que foi difícil?)

\begin{tabular}{|c|c|c|c|c|c|c|c|c|c|c|}
\hline \multirow{2}{*}{ O que foi difícil? } & \multirow{2}{*}{ Princípio } & \multirow{2}{*}{ Respostas } & \multicolumn{3}{|c|}{ Atividade $\mathrm{A}$} & & \multicolumn{3}{|c|}{ Atividade D } & \multirow[b]{2}{*}{ sig } \\
\hline & & & $\mathrm{N}$ & $\mathrm{EC}$ & $\mathrm{S}$ & & $\mathrm{N}$ & $\mathrm{EC}$ & $S$ & \\
\hline \multirow{2}{*}{ nada } & \multirow{2}{*}{$A, B, C, D$} & 24 & & 6 & 18 & 0,0227 & & 11 & 13 & 0,8388 \\
\hline & & & $0 \%$ & $25,00 \%$ & $75,00 \%$ & & $0 \%$ & $45,83 \%$ & $54,17 \%$ & \\
\hline \multirow{2}{*}{ bingo } & \multirow{2}{*}{$A, B, C, D$} & 12 & & 5 & 7 & 0,7744 & 4 & 4 & 4 & 1,000 \\
\hline & & & $0 \%$ & $41,67 \%$ & $58,33 \%$ & & $33,33 \%$ & $33,33 \%$ & $33,33 \%$ & \\
\hline \multirow{2}{*}{ jogo das cores } & \multirow{2}{*}{$A, B, C, D$} & 7 & & 1 & 6 & 0,1250 & 1 & 1 & 5 & 0,1017 \\
\hline & & & $0 \%$ & $14,29 \%$ & $85,71 \%$ & & $14,29 \%$ & $14,29 \%$ & $71,43 \%$ & \\
\hline \multirow{2}{*}{ jogo das mãos } & \multirow{2}{*}{$A, B, C, D$} & 6 & & 1 & 5 & 0,2188 & & 3 & 3 & 1,000 \\
\hline & & & $0 \%$ & $16,67 \%$ & $83,33 \%$ & & $0 \%$ & $50,00 \%$ & $50,00 \%$ & \\
\hline \multirow{2}{*}{ contar bolinhas } & \multirow{2}{*}{$A, B, D$} & 4 & & 3 & 1 & 0,6250 & & 1 & 3 & 0,6250 \\
\hline & & & $0 \%$ & $75,00 \%$ & $25,00 \%$ & & $0 \%$ & $25,00 \%$ & $75,00 \%$ & \\
\hline qual está & \multirow{2}{*}{$A, B$} & 7 & & 3 & 4 & 1,000 & & 3 & 4 & 1,000 \\
\hline faltando & & & $0 \%$ & $42,86 \%$ & $57,14 \%$ & & $0 \%$ & $42,86 \%$ & $57,14 \%$ & \\
\hline achar no no & \multirow{2}{*}{$A, B$} & 3 & & 2 & 1 & 1,000 & 1 & & 2 & 1,000 \\
\hline jornal & & & $0 \%$ & $66,67 \%$ & $33,33 \%$ & & $33,33 \%$ & $0,00 \%$ & $66,67 \%$ & \\
\hline
\end{tabular}

Fonte: elaborada pelas autoras. Legenda: "o que foi difícil" - pergunta realizada pela pesquisadora; "princípios" - princípios de contagem representados pelas letras; "atividade A" - correspondência termo a termo"; "atividade D" - irrelevância da ordem; "N"- princípio não construído; "EC" - princípio em construção; "S"- sim, princípio consolidado; sig - significância estatística. 
Novamente, nesta tabela, as atividades B e C não foram incluídas porque apresentaram a mesma distribuição de constructos das tabelas anteriores: todas as crianças com o constructo "S", exceto, neste caso, a resposta "nada" da atividade C. As crianças que responderam "nada" à pergunta "o que foi difícil" supostamente dominariam todos os princípios de contagem, não encontrando dificuldades em nenhuma tarefa da intervenção. De fato, os dados evidenciam que a maioria do grupo obteve constructos "EC" e "S" na tarefa avaliativa, com apenas 1 aluno não demonstrando consolidação do princípio abstração, sendo minoria significativa ( $p$ valor $<0,001)$.

\section{Discussão}

Os objetivos deste trabalho consistiam em investigar o conhecimento metacognitivo de alunos do $1^{\circ}$ ano do Ensino Fundamental em relação às sessões de intervenção em princípios de contagem que participaram e verificar se este conhecimento corresponderia ao desempenho dos sujeitos em avaliação dos princípios de contagem.

O conhecimento metacognitivo dos alunos foi averiguado através das perguntas realizadas pela pesquisadora. As categorias, correspondentes às respostas dos indivíduos, refletem os relatos sobre as sessões de intervenção e indicam, inicialmente, que as crianças tiveram clareza de apontar os aspectos solicitados (o que aprendeu, o que foi fácil e o que foi difícil).

É importante destacar que, em alguns casos, como as respostas "tudo" e "nada" às questões "o que foi fácil" e "o que foi difícil", respectivamente, podem ser indicativas de dois aspectos: primeiro, as crianças, de fato, não enfrentaram dificuldades nas tarefas da intervenção, até porque o grupo experimental foi composto, também, por alunos com alto desempenho nos princípios de contagem e que, por conta disso, provavelmente não manifestaram dificuldades; em segundo lugar, podem representar uma generalização, por parte dos sujeitos, ao não saber exatamente qual tarefa indicar, optando por uma resposta "global". 
No que diz respeito à correspondência entre o conhecimento metacognitivo dos estudantes e seu desempenho na avaliação em princípios de contagem, foi possível observar, de modo geral, que o relato da maioria dos alunos estava de acordo com sua performance na tarefa avaliativa. Ainda assim, foram identificados casos específicos em que as crianças afirmaram ter aprendido ou achado algo fácil/difícil e não tiveram estes aspectos confirmados no seu desempenho. Alguns exemplos: na tabela "o que aprendeu", 5 crianças disseram ter aprendido a contar do jeito certo, mas evidenciaram constructo "N" em alguns princípios; na tabela "o que foi fácil", novamente 5 alunos afirmaram ter achado as tarefas "jogo das cores", "jogo das mãos", "contar bolinhas" ou "tudo" fáceis e também obtiveram constructo "N" nos princípios de contagem; na tabela "o que foi difícil", 1 aluno afirmou não ter enfrentado dificuldade em nada e evidenciou constructo "N" em um princípio e houve alunos que afirmaram ter enfrentado dificuldades em vários princípios e, na verdade, foram bem no pós-teste.

As análises estatísticas confirmaram que as proporções das respostas foram diferentemente divididas $(p<0,05)$, apontando para associação entre constructo e categoria (resposta) em alguns casos. A categoria "contar do jeito certo", relativa à pergunta "o que foi fácil", apresentou proporções diferentes nas atividades C e D; as categorias "tudo" e "contar bolinhas", referentes à pergunta "o que foi fácil", apresentaram proporções diferentes nas atividades $C$ e $D$, respectivamente; a categoria "nada", relacionada à pergunta "o que foi difícil", teve proporções diferentes nas atividades $\mathrm{A}$ e $\mathrm{C}$.

É necessário interpretar estes dados com precaução, principalmente os resultados relacionados à pergunta "o que foi difícil?". Embora, por um lado, tenha sido observada certa discrepância entre os relatos dos alunos e seu desempenho no pós-teste, conforme mencionado anteriormente, é preciso chamar atenção para o fato de que a intervenção em princípios de contagem foi realizada justamente para auxiliar os alunos no processo de consolidação dos princípios. Embora alguns sujeitos, especificamente neste caso, tenham relatado dificuldades em algumas tarefas e, depois, apresentado bom desempenho na avaliação, isto indica que seu processo de aprendizagem ocorreu de forma 
positiva: uma dificuldade foi encontrada, como eles mesmos indicaram, porém, foi superada com a ajuda da intervenção.

De modo geral, os resultados encontrados apontam para a importância do conhecimento metacognitivo em relação ao desempenho matemático. Isto vai ao encontro de outros estudos que evidenciaram a relação entre o nível de conhecimento metacognitivo e o nível de conhecimento matemático (AUNOLA et al., 2004), destacando que alunos que apresentam boa performance em matemática também possuem boa performance metacognitiva (ÖZSOY, 2011). Nesse sentido, conforme discutido anteriormente e exposto na Figura 1 que apresenta as ideias de Garofalo e Lester (1985) e de Flavell (1979), tanto o conhecimento e crenças sobre o fenômeno cognitivo (representados pelo conhecimento metacognitivo sobre si, tarefas e estratégias) quanto o controle sobre as ações cognitivas (representado pelas experiências metacognitivas) possuem relação com a aprendizagem matemática e necessitam ser mais estudados e abordados em sala de aula.

Os dados aqui discutidos contribuem para a compreensão da necessidade do trabalho com a metacognição nas escolas, especialmente por conta da sua relação com a aprendizagem. Se o sujeito não possui conhecimento metacognitivo adequado a respeito de seu papel como aprendiz, das coisas que faz bem e de suas dificuldades, acaba por não ter consciência da necessidade de utilizar ou trocar determinadas estratégias para aprender efetivamente. Nesse sentido, tais aspectos de metacognição devem estar mais presentes nos processos formativos, visando informar $e$ instrumentalizar os professores para que possam favorecer a aprendizagem metacognitiva em sala de aula. A metacognição é parte essencial do aprender e precisa ser trabalhada desde cedo com os alunos, visando auxiliá-los a ter consciência de como aprendem, sendo participantes ativos deste processo.

\section{Limitações}

No que diz respeito ao referencial teórico, embora a área da metacognição seja estudada há muitas décadas, ainda existem muitos fatores sendo investigados e descobertos: estudiosos debruçam-se sobre a definição, avaliação, intervenção e, 
por mais que exista grande volume de produção na área, ainda há um longo caminho a ser percorrido. É importante destacar que poucos estudos são realizados com crianças pequenas e o referencial bibliográfico encontrado para este artigo consiste, em sua maioria, de trabalhos com alunos dos anos finais do Ensino Fundamental ou com universitários.

Os instrumentos utilizados neste trabalho também foram uma limitação. $A$ investigação do conhecimento metacognitivo foi realizada através de uma pequena entrevista, composta por apenas três perguntas, consistindo em um instrumento informal de pesquisa. Para realização de próximas pesquisas, contar com mais instrumentos, como questionários normatizados, por exemplo, pode fornecer mais informações acerca da metacognição.

Por fim, o tamanho da amostra (60 alunos) foi pequeno e isso pode ter impactado nas análises estatísticas. Por ter sido parte de uma amostra maior, participante de outro estudo, foi preciso trabalhar com este número de alunos, mas, para próximos estudos, aumentar o número de crianças investigadas poderá contribuir para diferentes resultados. É imprescindível ressaltar que a interpretação dos achados deste artigo deve ser realizada considerando o contexto em que foram produzidos.

\section{Conclusões e direções futuras}

A aprendizagem matemática é uma área de estudos que engloba muitos aspectos e a metacognição tem sido evidenciada como um fator chave neste processo. O estudo desenvolvido sugere que a maioria dos alunos relatou percepções sobre as tarefas de acordo com as dificuldades ou facilidades que encontraram nas sessões de intervenção, de modo que seu desempenho na avaliação em princípios de contagem tenha demonstrado confirmação dos relatos. Ainda assim, houve casos em que algumas crianças relataram determinada percepção e seu desempenho evidenciou outra, apontando para a necessidade de atentar para o conhecimento metacognitivo destes sujeitos. 
Esta pesquisa contribuiu para a discussão sobre a relevância da relação entre metacognição e matemática e destaca a importância de ajudar os alunos a utilizarem seus conhecimentos metacognitivos a seu favor, auxiliando-os na aquisição de novos conhecimentos e no monitoramento de suas aprendizagens.

O aprofundamento dos estudos nesta área possibilitará avanços nos processos de prevenção e intervenção das dificuldades na matemática. Investigações deste tipo possibilitam o conhecimento acerca dos possíveis obstáculos cognitivos e metacognitivos que dificultam determinadas aprendizagens para que, então, seja possível fazer frente a estes obstáculos por meio de seleção de recursos didáticos, conteúdos de ensino e estratégias de aprendizagem adequadas.

\section{Agradecimentos}

Agradecemos à Coordenação de Aperfeiçoamento de Pessoal de Nível Superior (CAPES) pelo financiamento para a realização desta pesquisa.

\section{Referências}

AUNOLA, Kaisa; LESKINEN, Esko; LERKKANEN, Marja-Kristiina; NURMI, JariErik. Developmental Dynamics of Math Performance From Preschool to Grade 2. Journal of Educational Psychology, v. 96, n. 4, p. 699-713, 2004.

CORSO, Helena Vellinho; SPERB, Tania Mara; JOU, Graciela Inchausti de; SALLES, Jerusa Fumagalli de; Metacognição e Funções Executivas: Relações entre os Conceitos e Implicações para a Aprendizagem. Psicologia: Teoria e Pesquisa, v. 29, n. 1, p. 21-29, 2013.

CORSO, Luciana Vellinho. Aprendizagem e desenvolvimento saudável:

Contribuições da Psicopedagogia. In: SANTOS, Bettina S. S.; ANNA, Lucia de. (Orgs.). Espaços psicopedagógicos em diferentes cenários [recursos eletrônico]. 1ed. Porto Alegre: EDIPUCRS, p. 64-76, 2013.

EKFLIDES, Anastasia; TSIORA, Aglaia. Metacognitive experiencies, self-concept and self-regulation. Psychologia, v. 25, p. 222-236, 2002. 
EFKLIDES, Anastasia; VLACHOPOULOS, Symeon P; Measurement of Metacognitive Knowledge of Self, Task, and Strategies in Mathematics. European Journal of Psychological Assessment, v. 28, n. 3, p. 227-239, 2012.

FLAVELL, John H. Metacognition and Cognitive Monitoring: A New-Area of Cognitive-Development Inquiry. American Pshychologist, v. 34, n. 10, p. 906911, 1979.

GAROFALO, Joe; LESTER, Frank K; Metacognition, cognitive monitoring, and mathematical performance. Journal for Research in Mathematics Education, v. 16, n. 3, p. 163-176, 1985.

ÖZSOY, Gökhan. An investigation of the relationship between metacognition and mathematics achievement. Asia Pacific Education Review, v. 12, n. 2, p.227235, 2010.

RIBEIRO, Célia. Metacognição: Um Apoio ao Processo de Aprendizagem.

Psicologia: Reflexão e Crítica, v. 16, n. 1, p. 109-116, 2003.

SCHNEIDER, Wolfgang; ARTELT, Cordula. Metacognition and mathematics education. Mathematics Education, v. 42, p. 149-161, 2010.

THRONDSEN, Inger. Self-regulated learning of basic-arithmetic skills: A longitudinal study. British Journal of Educational Psychology, v. 81, p. 558-578, 2011.

\section{Correspondência}

Évelin Fulginiti de Assis - Rua Ernesto Fontoura, número 343, apto 01, bairro São Geraldo, CEP 90230-091, Porto Alegre, Rio Grande do Sul, Brasil.

\section{Notas}

${ }^{1}$ O termo "universals of cognition" não foi traduzido por conta de sua complexidade conceitual.

2 "Avoidance" não foi traduzido por conta de sua complexidade conceitual, mas há indicação de possível entendimento no corpo do texto. 